\title{
Statistical Analysis of Single-Beam Interference Alignment Schemes
}

\author{
I. Santamaria, J. Fanjul \\ Dept. of Communications Engineering, University of Cantabria, Spain, \\ Email: \{nacho, jacobo\}@gtas.dicom.unican.es
}

\begin{abstract}
In this work, we derive analytical approximate expressions for the user rates achievable by interference alignment (IA) algorithms in single-beam multiple-input multiple-output (MIMO) networks for a fixed channel realization. Unlike previous works that perform a large-system analysis in which the number of users, antennas, or streams is required to tend to infinity, in this paper we only require that the number of different IA solutions (precoders and decoders) for the given scenario is sufficiently high, which typically happens even for moderate-size feasible networks. Based on the assumption that the IA beamformers for a given channel realization are random vectors isotropically distributed on the complex unit sphere, we characterize the user rates by averaging over the (possible finite) set of IA solutions. Some simulation results show the accuracy of the proposed rate expressions.
\end{abstract}

\section{INTRODUCTION}

Interference alignment (IA) has received a lot of attention in recent years as a key technique to achieve the maximum degrees of freedom (DoF) of wireless networks in the presence of interference. The basic idea of IA consists of designing the transmitted signals in such a way that the interference at each receiver falls within a lower-dimensional subspace, therefore leaving a subspace free of interference for the desired signal [1], [2], [3].

The goal of this paper is to analyze the performance of IA algorithms for the $K$-user multiple-input multiple-output (MIMO) interference channel when each user wishes to send a single beam or message. Due to the lack of analytical expressions for the rates achievable by interference alignment algorithms, the performance of IA schemes is typically characterized by means of Monte Carlo simulations. This might be a time consuming and computationally complex task, especially for scenarios involving a large number of users and/or antennas, for which no closed-form solution for the IA precoders exist and one has to resort to iterative optimization algorithms such as [4], [5], [6], [7].

The asymptotic performance of IA systems was first analyzed in [8] in the large-system limit in which the number of users and antennas tend to infinity at a fixed ratio. This analysis allowed the authors to derive approximations for the asymptote (i.e., the multiplexing gain or DoF) and offset of the sum-rate curve. More recently, random matrix theory was used in [9] to derive analytical rate expressions of IA schemes

This work was supported by the Ministerio de Economia y Competitividad (MINECO), Spain, under project RACHEL (TEC2013-47141-C4-3-R) and FPI grant BES-2014-069786. also in the large system limit in which the number of antennas and streams tend to infinity, but the number of users is fixed. More recently, the performance analysis of IA under imperfect channel state information and limited feedback models has been considered in [10], [11]. The goal of these works, however, is to analyze the throughput loss as a function of the number of bits used to quantize the channels and the IA precoding and decoding vectors.

In this work, we derive analytical approximate expressions for the per-user rates achievable by IA algorithms in singlebeam MIMO networks for a fixed channel realization. Instead of performing a large-system analysis in which the number of users, antennas, or streams is required to grow, we only require that the number of different IA solutions for the given scenario is sufficiently high, which typically happens for moderate size networks [12], [13].

The analysis is based on the assumption (supported by numerical results) that, for a given channel realization, the precoding and decoding vectors corresponding to different alignment solutions are random vectors isotropically distributed on the complex unit sphere. Building on this assumption, we characterize the performance of single-beam IA schemes by averaging over the (possible finite) set of IA solutions, instead of the more traditional approach that averages over the random channel realizations. Specifically, we characterize the signal-to-noise ratio of a given user as a scaled beta distribution, which allows us to derive analytical expressions for the rate achievable by a particular user when either a random IA solution or the best-out-of-L solution is applied. We also provide approximate expressions for the average sum-rate achievable by the $\mathrm{K}$ users. In the end, this analysis will help us to answer the question: How many IA solutions should we compute to obtain a performance close to that of the maximum achievable sum-rate solution?

\section{A. Notation}

In this paper we use bold-faced upper case letters to denote matrices, bold-faced lower case letters for column vectors, and light-face lower case letters for scalar quantities. The superscript $(\cdot)^{H}$ denotes Hermitian. The Frobenius norm of a matrix $\mathbf{A}$ will be denoted as $\|\mathbf{A}\|_{F}$, and the Euclidean norm of a vector is $\|\mathbf{x}\| . \mathbf{u} \otimes \mathbf{v}$ is the Kronecker product of vectors $\mathbf{u}$ and $\mathbf{v}$, and $\operatorname{vec}(\mathbf{H})$ is a vector obtained by stacking the columns of $\mathbf{H}$ on top of one another. Finally, the notation 
$\mathbf{x} \in \mathbb{S}_{1}\left(\mathcal{C}^{N}\right)$ denotes that $\mathbf{x}$ belongs to the Stiefel manifold of unit-norm vectors in $\mathcal{C}^{N}$.

\section{Problem Statement}

We consider a $K$-user multiple-input multiple-output (MIMO) interference channel with constant channel coefficients, where each user wishes to send a single data stream using IA [1], [2]. For simplicity, we focus on a symmetric scenario in which each transmitter is equipped with $M$ antennas and each receiver has $N$ antennas. Following the conventional notation [14], we denote this single-beam MIMO network as $(M \times N, 1)^{K}$.

The MIMO channel from transmitter $l$ to receiver $k$ is denoted as $\mathbf{H}_{k l}$ and assumed to be flat-fading and constant over time. Each $\mathbf{H}_{k l}$ is an $N \times M$ complex matrix with independent entries drawn from a continuous distribution. We also assume that the IA problem is feasible, which can be checked for some particular scenarios using the theoretical results in [14][16] or, for arbitrary networks, with the polynomial complexity feasibility test proposed in [17].

For feasible systems, it has been recently proved in [12], [13] that the number of different alignment solutions for a given channel realization may be either finite (when $M+N=$ $K+1$ ) or infinite (when $M+N>K+1$ ). Furthermore, for single beam systems the exact number of solutions can be easily obtained by the combinatorial counting procedure described in [13].

We now introduce some notation that will be needed in the subsequent analysis. The $i$-th alignment solution is formed by unit-norm precoders and decoders on the complex Stiefel manifolds: $\mathbf{v}_{k, i} \in \mathbb{S}_{1}\left(\mathcal{C}^{M}\right)$ and $\mathbf{u}_{k, i} \in \mathbb{S}_{1}\left(\mathcal{C}^{N}\right)$, which satisfy the IA equations

$$
\begin{aligned}
\mathbf{u}_{k, i}^{H} \mathbf{H}_{k l} \mathbf{v}_{l, i} & =0, \quad \forall k \neq l \\
\mathbf{u}_{k, i}^{H} \mathbf{H}_{k k} \mathbf{v}_{k, i} & \neq 0 .
\end{aligned}
$$

The $i$-th IA solution for the $K$ users is collectively denoted as $\mathcal{S}^{i}=\left\{\left(\mathbf{v}_{k, i}, \mathbf{u}_{k, i}\right), k=1, \ldots, K\right\}$ and the set of all IA solutions is denoted as $\mathcal{S}=\left\{\mathcal{S}^{i}, i=1, \ldots, P\right\}$, with $P \in \mathbb{N}^{+}$ being the total number of different IA solutions. Notice that, due to the problem invariances, if $\mathbf{v}_{k, i}$ is a solution of the IA problem, then $\mathbf{v}_{k, i} e^{j \theta}$ is also a solution. In fact, from the point of view of this analysis $\mathbf{v}_{k, i}$ and $\mathbf{v}_{k, i} e^{j \theta}$ are exactly the same solution: i.e., the $P$ solutions are counted in the Grassmann manifold.

Our main goal is to derive approximate expressions for the average rate achievable by user $k$ when a random IA solution (or the best-out-of-L solution) from the set $\mathcal{S}$ is used:

$$
\mathrm{E}_{\mathcal{S}}\left[C_{k}\right]=\mathrm{E}_{\mathcal{S}}\left[\log \left(1+\frac{P_{k}\left|\mathbf{u}_{k, i}^{H} \mathbf{H}_{k k} \mathbf{v}_{k, i}\right|^{2}}{\sigma_{k}^{2}}\right)\right],
$$

where $P_{k}$ is the transmitted power of user $k$ and $\sigma_{k}^{2}$ is the variance of the additive white Gaussian noise at the input of the $k$-th receiver. Notice that, unlike most works dealing with the statistical characterization of wireless communication systems, in (3) the expectation is not computed over the distribution of the channel coefficients, but over the (possibly finite) set $\mathcal{S}$ of IA solutions for the $k$ user. To stress this point we denote the mathematical expectation as $\mathbf{E}_{\mathcal{S}}$.

\section{Statistical AnAlysis}

For a feasible system, the signal-to-noise-ratio (SNR) after perfect IA at receiver $k$ can be expressed as

$$
\begin{aligned}
\mathrm{SNR}_{k} & =\frac{P_{k}\left|\mathbf{u}_{k, i}^{H} \mathbf{H}_{k k} \mathbf{v}_{k, i}\right|^{2}}{\sigma_{k}^{2}} \\
& =\frac{P_{k}|| \mathbf{h}_{k k}||^{2}\left|\left(\mathbf{v}_{k, i}^{*} \otimes \mathbf{u}_{k, i}\right)^{H} \cdot \tilde{\mathbf{h}}_{k k}\right|^{2}}{\sigma_{k}^{2}},
\end{aligned}
$$

where

$$
\mathbf{h}_{k k}=\operatorname{vec}\left(\mathbf{H}_{k k}\right)=\left\|\mathbf{h}_{k k}\right\| \tilde{\mathbf{h}}_{k k} .
$$

Let us denote $\mathbf{b}_{k, i}=\mathbf{v}_{k, i}^{*} \otimes \mathbf{u}_{k, i}$. Since the design of the IA precoders and decoders does not involve the direct channel matrix $\mathbf{H}_{k k}, \mathbf{b}_{k, i}$ is a unit-norm $M N \times 1$ column vector independent of $\tilde{\mathbf{h}}_{k k}$.

\section{A. Isotropically distributed IA solutions}

In this work, we make the following main assumption:

(A1) For a given channel realization, the vectors $\mathbf{b}_{k, i}$ corresponding to different IA solutions are independent and isotropically distributed on the $M N$-dimensional complex unit sphere.

To check the validity of this assumption we consider the interference channel $(2 \times 5,1)^{6}$, for which there are a total of $P=265$ different alignment solutions [13]. We obtained all 265 solutions using the procedure described in [18], and computed the pairwise angle between vectors $\mathbf{b}_{k, i}$ and $\mathbf{b}_{k, j}$ corresponding to two different solutions. Fig. 1 compares the estimated cumulative distribution function (cdf) for the pairwise angle with the theoretical cdf of isotropically generated random vectors [19]. We can observe that, for feasible systems and when the number of solutions is sufficiently high, the isotropic assumption is a rather accurate approximation for the distribution of IA solutions.

To further validate the isotropic assumption, let $\mathbf{b}_{k, i}, i=$ $1, \ldots, n \leq P$ be a random sample from the set of $P$ different IA solutions. Without any loss of generality, we assume that the real random vectors formed by stacking the real and imaginary parts of $\mathbf{b}_{k, i}$ have been drawn according to a $2 M N$-variate von Mises-Fisher distribution [20]: $\left(\mathfrak{R}\left(\mathbf{b}_{k, i}\right), \mathfrak{I}\left(\mathbf{b}_{k, i}\right)\right) \sim \mathcal{M}(\mu, \kappa)$, where $\mu$ indicates the direction along which the unit-norm vectors are clustered and $\kappa$ is the concentration parameter. The greater is $\kappa$, the greater the clustering around the mean direction given by $\mu$; whereas for $\kappa=0$ the distribution is isotropic. Now, given $\mathbf{b}_{k, i}, i=$ $1, \ldots, n \leq P$, we consider the following binary hypothesis testing:

$$
\begin{aligned}
& H_{0}: \kappa=0 \\
& H_{1}: \kappa \neq 0,
\end{aligned}
$$




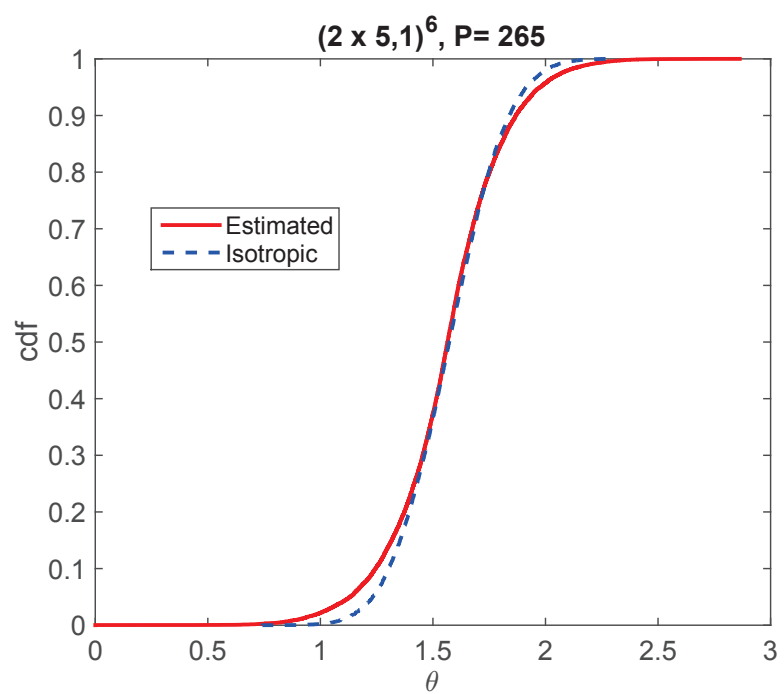

Fig. 1. Comparison of the pairwise angle cdf between two different IA solutions for the $(2 \times 5,1)^{6}$ MIMO-IC. The system has a total of $P=265$ IA solutions.

where the direction parameter $\mu$ is unknown. The uniformly most powerful invariant test (UMPIT) for this problem is the Rayleigh test, which is given by [20], [21]

$$
\left\|\overline{\mathbf{b}}_{k}\right\|^{2} \underset{H_{0}}{\stackrel{H_{1}}{\gtrless}} \eta
$$

where the threshold $\eta$ is fixed for a given probability of false alarm, and $\overline{\mathbf{b}}_{k}=\frac{1}{n} \sum_{i=1}^{n} \mathbf{b}_{k, i}$. We run the test for 100 different $(2 \times 5,1)^{6}$ MIMO-IC channels. More precisely, we computed all $P=265$ IA solutions for each channel realization, and used all solutions in the test $(n=P)$. For a $P_{f a}=0.1$, the test always accepted the null hypothesis, thus validating the isotropic assumption. The same result was obtained for other systems with more IA solutions.

\section{B. Achievable rates for a random IA solution}

Our first goal is to characterize statistically the SNR for user $k$, given by (4), when its precoder-decoder pair is taken at random from the set of $P$ different IA solutions. According to Assumption $1, \mathbf{b}_{k, i}=\mathbf{v}_{k, i}^{*} \otimes \mathbf{u}_{k, i}$ is isotropically distributed in $\mathbb{S}_{1}\left(\mathcal{C}^{M N}\right)$

$$
p\left(\mathbf{b}_{k, i}\right)=\frac{\Gamma(M N)}{2 \pi^{M N}} \delta\left(\mathbf{b}_{k, i}^{H} \mathbf{b}_{k, i}-1\right),
$$

where $\Gamma(n)=(n-1)$ ! denotes the Gamma function. We have the following result.

Proposition 1. The random variable $\mathrm{SNR}_{k}$ can be expressed as,

$$
\mathrm{SNR}_{k}=\rho_{k} X
$$

where $\rho_{k}=\frac{P_{k}\left\|\mathbf{H}_{k k}\right\|_{F}^{2}}{\sigma_{k}^{2}}$ is a deterministic constant, and $X$ is beta distributed with parameters 1 and $M N-1$, that is, $X \sim \operatorname{Beta}(1, M N-1)$.
Proof. From (4), we see that $X=\left|\mathbf{b}_{k, i}^{H} \tilde{\mathbf{h}}_{k k}\right|^{2}$. Taking into account that $\tilde{\mathbf{h}}_{k k}$ is a fixed unit norm vector that points in a direction determined by the direct link MIMO channel, we see that $X$ is the squared length of the projection of $\tilde{\mathbf{h}}_{k k}$ onto a vector isotropically distributed on the $M N$-dimensional complex hypersphere, which is known to follow a beta distribution [22], [23], [24], and thus $X \sim \operatorname{Beta}(1, M N-1)$.

Since $\mathrm{SNR}_{k}$ follows a scaled beta distribution, the average rate achievable by user $k$ when a random IA solution from the set $\mathcal{S}$ is used is given by the following expression [25]

$$
\begin{aligned}
& \mathrm{E}_{\mathcal{S}}\left[C_{k}\right]=\mathrm{E}_{\mathcal{S}}\left[\log \left(1+\mathrm{SNR}_{k}\right)\right] \\
& \quad\left(\frac{\log (e) \rho_{k}}{M N}\right){ }_{3} F_{2}\left(1,1,2 ; 2, M N+1 ;-\rho_{k}\right),
\end{aligned}
$$

where ${ }_{3} F_{2}\left(a_{1}, a_{2}, a_{3} ; b_{1}, b_{2} ; x\right)$ denotes the generalized hypergeometric function. Finally, using (7), the average sum-rate for the $K$ users can be easily obtained as

$$
\mathrm{E}_{\mathcal{S}}[C]=\frac{1}{K} \sum_{k=1}^{K} \mathrm{E}_{\mathcal{S}}\left[C_{k}\right]
$$

\section{Achievable rates for the best-out-of-L IA solution}

Suppose we obtain now $L$ different solutions (out of the total $P$ ) and use the best one for user $k$. Then, the signal-tonoise ratio for user $k$ is

$$
\operatorname{SNR}_{k, L}=\rho_{k} \max \left(X_{1}, X_{2}, \cdots, X_{L}\right)=\rho_{k} Y,
$$

where $X_{l} \sim \operatorname{Beta}(1, M N-1)$ are i.i.d. betadistributed random variables, and we have defined $Y=\max \left(X_{1}, X_{2}, \cdots, X_{L}\right)$. In this case, deriving a closed-form expression for the achievable rate does not appear to be feasible. Therefore, we apply Jensen's inequality and proceed as follows

$$
\mathrm{E}_{\mathcal{S}}\left[C_{k}^{L}\right] \leq \log \left(1+\rho_{k} \mathrm{E}_{\mathcal{S}}[Y]\right),
$$

where $C_{k}^{L}$ denotes the average sum-rate achievable by user $k$ when the best-out-of-L alignment solution is used. The expectation of the maximum of $L$ i.i.d beta-distributed variables can be obtained as follows

$$
\mathrm{E}[Y]=\int_{0}^{1}(1-F(Y)) d y=\int_{0}^{1}\left(1-F(X)^{L}\right) d x
$$

where $F(X)=1-(1-x)^{M N-1}$ is the distribution function of $X \sim \operatorname{Beta}(1, M N-1)$. The expectation in (10) is given by ${ }^{1}$

$$
\mathrm{E}[Y]=\sum_{l=1}^{L}\left(\begin{array}{l}
L \\
l
\end{array}\right)(-1)^{L+1} \frac{1}{l(M N-1)+1},
$$

and therefore, the proposed approximation for the expected achievable rate is

$$
\mathrm{E}_{\mathcal{S}}\left[C_{k}^{L}\right]=\log \left(1+\rho_{k} \sum_{l=1}^{L}\left(\begin{array}{l}
L \\
l
\end{array}\right)(-1)^{L+1} \frac{1}{l(M N-1)+1}\right) \text {. }
$$

\footnotetext{
${ }^{1}$ For values of $L>100$, some numerical problems may appear when computing very large combinatorial numbers. For these cases, it is better to directly perform the numerical integration of (10).
} 
Remark: It can be shown that

$$
\begin{aligned}
\sum_{l=1}^{L}\left(\begin{array}{l}
L \\
l
\end{array}\right)(-1)^{L+1} \frac{1}{l(M N-1)+1} \leq \\
\frac{1}{M N-1} \sum_{l=1}^{L}\left(\begin{array}{l}
L \\
l
\end{array}\right)(-1)^{L+1} \frac{1}{l}=\frac{\mathcal{H}_{L}}{M N-1},
\end{aligned}
$$

where $\mathcal{H}_{L}$ is the Harmonic number. On the other hand, for large $L, \mathcal{H}_{L} \approx \gamma+\ln (L)$, where $\gamma$ is the Euler-Mascheroni constant. This result indicates that the SNR for user $k$ increases only logarithmically with the number of explored IA solutions, and hence exploring a small percentage of solutions provides most of the benefit.

Obviously, the best IA solution for user $k$ is not necessarily the best solution for the rest of users. To derive an expression for the sum-rate when the best average solution for the whole network is used we need yet another approximation. We start by making the crude assumption that $\log \left(1+\rho_{k} X\right) \sim$ $N\left(\mu_{k}, \sigma_{k}^{2}\right)$, where $\mu_{k}$ is given by (7) and for the variance we use a first-order Taylor series expansion of the log function around its mean, that is

$$
\sigma_{k}^{2}=\left(\frac{\rho_{k} \log (e)}{M N+\rho_{k}}\right)^{2} \frac{M N-1}{M N+1} .
$$

Since the rates achieved by each user are assumed to be independent Gaussian variables, the average sum-rate when a random IA solution is applied is also Gaussianly distributed

$$
C=\frac{1}{K} \sum_{k=1}^{K} C_{k} \sim N\left(\frac{1}{K} \sum_{k=1}^{K} \mu_{k}, \frac{1}{K^{2}} \sum_{k=1}^{K} \sigma_{k}^{2}\right) .
$$

Finally, to characterize the average sum-rate for the bestout-of-L solution, when use the following approximation for expected value of the maximum of $L$ i.i.d. Gaussian random variables.

Proposition 2. Let $X_{1}, X_{2}, \ldots, X_{L}$ be $L$ i.i.d. Gaussian random variables with density function $X_{l} \sim N\left(\mu, \sigma^{2}\right)$, and let $Y=\max \left(X_{1}, X_{2}, \cdots, X_{L}\right)$. The expected value of $Y$ can be bounded as

$$
E[Y] \leq \mu+\sigma \sqrt{2 \ln (L)}
$$

Proof. For any $s>0$, we can apply Jensen's inequality to $e^{s Y}$ and obtain

$$
\begin{aligned}
e^{s \mathrm{E}[Y]} & \leq \mathrm{E}\left[e^{s Y}\right]=\mathrm{E}\left[\max _{l} e^{s X_{l}}\right] \\
& \leq \sum_{l=1}^{L} \mathrm{E}\left[e^{s X_{l}}\right]=L\left(e^{\mu s+\frac{s^{2} \sigma^{2}}{2}}\right),
\end{aligned}
$$

where the last equality follows from the moment generating function of $N\left(\mu, \sigma^{2}\right)$. Taking logarithms we can rewrite the above inequality as

$$
\mathrm{E}[Y] \leq \frac{\ln (L)}{s}+\mu+\frac{s \sigma^{2}}{2} .
$$

Now, we can set $s=\sigma \sqrt{2 \ln (L)}$ and the bound in (14) follows.
Finally, the proposed approximation for the average sumrate of the best-out-of- $\mathrm{L}$ solution is

$$
\mathrm{E}_{\mathcal{S}}\left[C^{L}\right]=\frac{1}{K}\left(\sum_{k=1}^{K} \mu_{k}+\sqrt{2 \ln (L) \sum_{k=1}^{K} \sigma_{k}^{2}}\right)
$$

where $\mu_{k}$ is given by (7) and $\sigma_{k}^{2}$ is given by (13).

\section{Simulation Results}

In this section we evaluate the accuracy of the per-user rate approximations given by (7) and (12), as well as their average sum-rate counterparts (8) and (15) by means of computer simulations. In the first example, we consider a single beam MIMO-IC with $K=6$ users, $M=2$ transmit antennas and $N=5$ receive antennas, for which a total of $P=265$ different IA solutions exist [13], and obtain a random IA solution using any of the algorithms proposed to this end [4][7]. Fig. 2 compares the true rate achieved by user 5 after alignment with the approximation given by (7), as well as the true and approximated average sum-rate for the 6 users. We observe that the proposed analytical rate expressions provide very accurate approximations for the true rates.

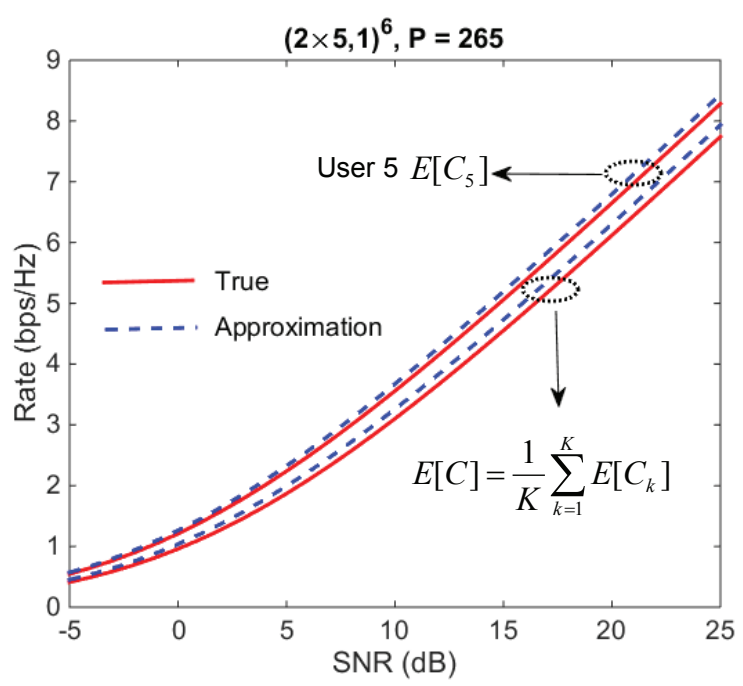

Fig. 2. Comparison of the true rates with the approximations given by (7) and (8) when a random alignment solution is used. The scenario is a $(2 \times 5,1)^{6}$ MIMO-IC, which has $P=265$ solutions.

In the second example, we consider the network $(3 \times 4,1)^{6}$ which has $P=7570$ IA solutions [13]. For a given channel realization we obtain a subset of solutions by initializing the interference leakage minimization algorithm at multiple random points. The signal-to-noise-ratio for this example has been fixed to SNR $=15 \mathrm{~dB}$. Fig. 3 depicts the rate achieved by user 3 when the best-out-of-L alignment solution is used as a function of $\mathrm{L}$, and compare it with the approximation given by (12). Also, the true average sum-rate is compared with the approximation in (15). We observe that both approximations capture quite well the logarithmic behavior of the true curves and, in general, the approximations are very accurate. For this 
scenario, by selecting the best out of 25 IA solutions, which is less than $0.4 \%$ of the total 7570 solutions, we get more than $90 \%$ of the maximum rate for this scenario. This behavior has been corroborated in many other scenarios, allowing us to conclude that exploring only a tiny fraction of the total number of solutions is sufficient to operate close to the optimal performance.

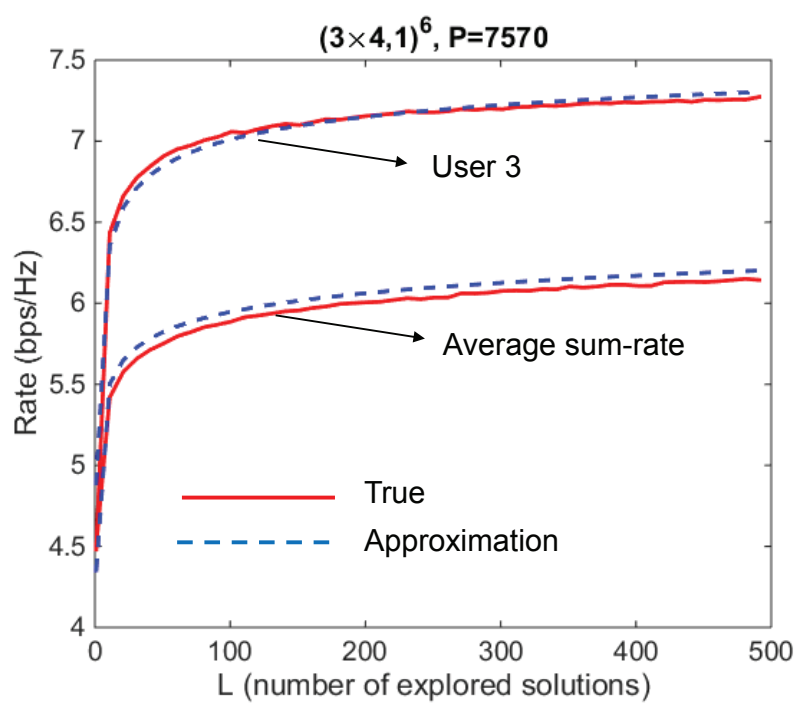

Fig. 3. Comparison of the true rates with the approximations given by (12) and (15) when the best-out-of-L alignment solution is used. The scenario is a $(3 \times 4,1)^{6}$ MIMO-IC, which has $P=7570$ solutions, and the $\mathrm{SNR}=15$ dB.

\section{CONCLUSIONS}

This paper derived analytical approximate rate expressions for interference alignment schemes in single-beam MIMO networks. The main assumptions were that the number of IA solutions for a given channel realization is sufficiently high, and that these solutions are isotropically distributed random vectors on the Stiefel manifold. The obtained expressions allow us to predict rather accurately the performance of IA techniques without resorting to time consuming Monte Carlo simulations. Moreover, our analysis also revealed that the SNR for a particular user increases only logarithmically with the number of solutions, and hence finding only a small fraction of the total number of solutions is sufficient to achieve close to optimal performance. In future work, we plan to extend this analysis to multibeam IA networks, as well as to compare the proposed approximations with those derived in the largesystem limit.

\section{REFERENCES}

[1] S. A. Jafar, "Interference Alignment: A New Look at Signal Dimensions in a Communication Network," Foundations and Trends in Communications and Information Theory, vol. 7, no. 1, pp. 1-134, 2010.

[2] V. R. Cadambe, S. A. Jafar, "Interference alignment and degrees of freedom of the K-user interference channel," IEEE Trans. on Inf. Theory, vol. 54, no. 8, pp. 3425-3441, Aug. 2008.
[3] M. Maddah-Ali, A. Motahari, A. Khandani, "Communications over MIMO X channels: Interference alignment, decomposition, and performance analysis," IEEE Trans. on Inf. Theory, vol. 54, no. 8, pp. 34573470, Aug. 2008

[4] K. S. Gomadam, V. R. Cadambe, S. A. Jafar, "A distributed numerical approach to interference alignment and applications to wireless" interference networks, IEEE Trans. on Inf. Theory, vol. 57, no. 6, pp. 3309-3322, Jun. 2011.

[5] S. W. Peters, R. W. Heath, "Interference alignment via alternating minimization," IEEE Int. Conf. on Acoustics, Speech and Signal Proc. (ICASSP 2009), pp. 2445-2448, Taipei, Taiwan, Apr. 2009.

[6] H. Sung, S. H. Park, K. J. Lee, I. Lee, "Linear precoder designs for Kuser interference channels," IEEE Trans. on Wireless Communications, vol. 9, no. 1, pp. 291-301, Jan. 2010.

[7] O. Gonzalez, C. Lameiro, and I. Santamaria, "A quadratically convergent method for interference alignment in MIMO interference channels," IEEE Signal Proc. Letters, vol. 21, no. 11, pp. 1423-1427, Nov. 2014.

[8] D. A. Schmidt, W. Utschick, M. L. Honig, "Large system performance of interference alignment in single-beam MIMO networks," Proceedings of the IEEE Global Telecommunications Conference, (GLOBECOM), Miami, USA, 2010.

[9] S. Bazzi, G. Dietl, W. Utschick, "Large system analysis of interference alignment achievable rates for the MIMO interference channel," IEEE Trans. on Signal Processing, vol. 63, no. 6, pp. 1490-1499, 2015.

[10] H. Wang, R. Song, S.H. Leung, "Throughput analysis of interference alignment for a general centralized limited feedback models," IEEE Trans. on Vehicular Technology, DOI: 10.1109/TVT.2015.2509501, Dec. 2015.

[11] X. Chen, C. Yuen, "On interference alignment with imperfect CSI: Characterizations of outage probability, ergodic rate and SER," IEEE Trans. on Vehicular Technology, vol. 65, no. 1, pp. 47-58, Jan. 2016.

[12] O. Gonzalez, I. Santamaria, C. Beltran, "Finding the number of feasible solutions for linear interference alignment problems," IEEE Int. Symp. on Information Theory, Istanbul, Turkey, pp. 384-388, July, 2013.

[13] O. Gonzalez, C. Beltran, I. Santamaria, "On the number of interference alignment solutions for the K-User MIMO channel with constant coefficients," IEEE Trans. on Inf. Theory, vol.61, no. 11, pp. 6028-6048, Nov. 2015.

[14] C. M. Yetis, T. Gou, S. A. Jafar, A. H. Kayran, "On feasibility of interference alignment in MIMO interference networks," IEEE Trans. on Signal Processing, vol. 58, no. 9, pp. 4771-4782, Sept. 2010.

[15] M. Razaviyayn, G. Lyubeznik, Z.-Q. Luo, "On the degrees of freedom achievable through interference alignment in a MIMO interference channel," IEEE Trans. on Signal Processing, vol. 60, no. 2, pp. 812-821, 2012.

[16] L. Ruan, V. K. N. Lau, M. Z. Win, "The feasibility conditions for interference alignment in MIMO networks," IEEE Trans. on Signal Processing, vol. 61, no. 8, pp. 2066-2077, Apr. 2013.

[17] O. Gonzalez, C. Beltran, I. Santamaria, "A feasibility test for linear interference alignment in MIMO channels with constant coefficients," IEEE Trans. on Inf. Theory, vol. 60, n. 3, pp. 1840-1856, March, 2014.

[18] O. Gonzalez, I. Santamaria, "Interference alignment in single-beam MIMO networks via homotopy continuation," IEEE Int. Conf. on Acoustics, Speech and Signal Proc. (ICASSP 2011), Prague, Czech Republic, May, 2011.

[19] T. Cai, J. Fan, T. Jiang, "Distributions of angles in random packing on spheres," Journal of Machine Learning Research, vol. 14, pp. 18371864, 2013.

[20] K. V. Mardia, J. T. Kent, J. M. Bibby, Multivariate Analysis, Academic Press, 1979

[21] Y. Chikuse, Statistics on Special Manifolds, Lectures Notes on Statistics, Springer-Verlag, New York, 2003.

[22] M. L. Eaton, "On the projection of isotropic distributions," The Annals of Statistics, vol. 9, no. 2, pp. 391-400, 1981.

[23] S. D. Howard, S. Sirianunpiboon, D. Cochran, "Invariance of the distributions of normalized Gram matrices," IEEE Workshop on Statistical Signal Proc. (SSP 2014), Gold Coast, Australia, 2014.

[24] L. Sun, M. R. McKay, M. Lei, "Tomlinson-Harashima precoding for multiuser MIMO systems with quantized CSI feedback and user scheduling," IEEE Trans. on Signal Processing, vol. 62, no. 16, pp. 4077-4090, Aug., 2014

[25] A. K. Gupta, S. Nadarajah, Handbook of Beta Distribution and Applications, Marcel Dekker, New York, 2004. 